\title{
Evaluation of the Diagnostic Value of Digital Rectal Examination for Lymph Node Metastasis in Mid-Low Rectal Cancer
}

\author{
Yingai Jin1, Chunyu Li2*, Xian Zhang1* \\ ${ }^{1}$ Department of General Surgery, Affiliated Hospital of Yanbian University, Yanji, China \\ ${ }^{2}$ School of Nursing, Yanbian University, Yanji, China \\ Email: *chyli@ybu.edu.cn, *3060117457@qq.com
}

How to cite this paper: Jin, Y.A., Li, C.Y. and Zhang, X. (2019) Evaluation of the Diagnostic Value of Digital Rectal Examination for Lymph Node Metastasis in Mid-Low Rectal Cancer. International Journal of Clinical Medicine, 10, 317-325.

https://doi.org/10.4236/ijcm.2019.105024

Received: November 20, 2018

Accepted: May 6, 2019

Published: May 9, 2019

Copyright $\odot 2019$ by author(s) and Scientific Research Publishing Inc. This work is licensed under the Creative Commons Attribution International License (CC BY 4.0).

http://creativecommons.org/licenses/by/4.0/

\begin{abstract}
Background: NCCN's guidelines for the diagnosis and treatment of rectal cancer suggest that accurate preoperative clinical staging of rectal cancer is very important. Reliable preoperative evaluation is the key to the development of surgical protocols, in order to investigate the diagnostic value of digital rectal diagnosis for lymph node metastasis of middle and low rectal cancer. Methods. We prospectively performed digital rectal examination in 258 patients with mid-low rectal cancer before operation, to analyze the distance from the lower margin of the tumor to the margin of the anus, the diameter of the invasion of the intestinal wall of the tumor, the accuracy between the general type and depth of invasion of the tumor and the pathological results of the postoperative specimen, and the predictability of the lymph node metastasis rate of the rectal digital examination. Results. The results of the analysis showed that the above indicators and postoperative pathological findings have high accuracy. Conclusions. It is concluded that accurate and detailed digital rectal examination before operation can predict lymph node metastasis rate of mid-low rectal cancer at a higher level and accurately.
\end{abstract}

\section{Keywords}

Digital Rectal Examination, Rectal Cancer, Mid-Low, Lymphatic Metastasis

\section{Introduction}

The use of total mesorectal resection (TME) has become increasingly widespread, leading to a dramatic decrease in the prevalence of local recurrence of rectal cancer from $38 \%$ to less than 10\% [1]. Even with TME, however, the presence of a tumor or malignant node within $1 \mathrm{~mm}$ of the CRM remains an impor- 
tant predisposing factor for local recurrence [2]. NCCN's guidelines for the diagnosis and treatment of rectal cancer believe that accurate preoperative clinical staging of rectal cancer is very important. Preoperative simultaneous neoadjuvant therapy is the mode of treatment for stage II, III colorectal cancer patients [3]. The determination of the best surgical procedure for rectal cancer depends on an accurate preoperative assessment of the local progression and distant metastasis of rectal cancer. With the development of neoadjuvant therapy and surgical technique, the recurrence rate is obviously reduced, and the survival rate is now over 70\% [4]. Randomized trials have shown that combined preoperative radiation therapy-TME reduces the prevalence of local recurrence from $8 \%$ to $2 \%$ and is superior to postoperative radiation therapy alone [5] [6]. Therefore, reliable preoperative evaluation is crucial to the surgical plan. There are many imaging methods for preoperative clinical staging of rectal cancer, including MRI, PET, and CT [7]. However, the accuracy of imaging depends on the experience of the radiologist and there are significant internal observer differences [8] [9]. Endoscopic ultrasound (EUS) is reported to be a more accurate staging method [9] [10]. However, not all studies have confirmed the superiority of the United States [11]. Imaging studies rely mainly on lymph node size to assess lymph node metastases. The accuracy of simply determining lymph node metastasis based on lymph node size is still low. This is mainly because imaging does not easily identify reactive growth and lymph node metastasis [12]. The results showed that the accuracy of EUS, CT and MRI in lymph node metastasis was only $62 \%-87 \%, 22 \%-73 \%$ and $39 \%-75 \%$ [13]. Overall, image judgment is a process of visual perception and requires considerable experience.

The digital rectal examination can not only determine the presence of mid-low rectal cancer, but also can further understand the position, size, texture, degree of involvement and relationship of prostate, urethra, vagina and uterus. A total of 258 patients with low rectal cancer were enrolled in this study. The accuracy of palpation for preoperative assessment of lymph node metastasis in low-grade rectal cancer was assessed by digital rectal examination.

\section{Material and Methods}

\subsection{Patient Characteristics}

From January 2005 to December 2015, 258 cases of rectal cancer in general surgery department of Yanbian University affiliated Hospital were collected. Each patient was confirmed by fiberoptic colonoscopy and biopsy and surgically resected. This study excluded patients whose lesions could not be resected and whose preoperative anal finger examination was more than $8 \mathrm{~cm}$ from the anal margin. The study was approved by the Ethics Committee of Yanbian University affiliated Hospital. There were 97 males and 161 females, with an average age of $66 \pm 3.1$ years, the youngest age being 29 years and the maximum age 84 years. Digital rectal examination mainly assessed the distance from the lower margin of the tumor to the anal margin, the length of the diameter of the infiltrating intes- 
tinal wall, the gross pathological type, and the depth of the invasion. The patient is placed in the chest and knee position, Place the non-examining hand on the patient's right pelvic bone to provide counter-traction. Place your lubricated index finger on to the anus, pointing anteriorly and apply gentle pressure to the midline of the anus. Slowly enter the anus as you maintain pressure. After a few seconds, the anal sphincter should relax, allowing the digit to be advanced further into the rectum. Sweep your finger in a clockwise and then anti-clockwise manner to assess the entire circumference of the rectum. Feel for masses within the rectal wall (noting the approximate size, consistency and location-anterior, posterior, right or left lateral walls), note the approximate distance from the anal verge. If necessary, the patient squats, and increases abdominal pressure. The depth of invasion is as follows: intramucous carcinoma, soft lesion, no contact with hard parts, excellent mobility, almost not fixed in the rectal wall, free movement, difficult to distinguish from adenoma, a part of the tumor is hard and tough to infiltrate into the submucous layer. Submucosal carcinoma: because of the proliferation of submucosal fibrous tissue, compared with mucosal carcinoma, the texture becomes hard and tough, and the mobility is almost the same as mucosal carcinoma, moving with the mucosa, not with the intestinal wall. Carcinoma that infiltrates deep muscle layer: the quality is hard; the activity is restricted to some extent; the intestinal wall moves with the tumor. Immersion of deep muscle layer into extra intestinal fat: further reduction of activity. When adhesions infiltrate into other organs (prostate, vagina, presacral, etc.), tumor fixation and loss of activity. The specimens were examined by H-E staining under light microscope.

\subsection{Surgical Technique}

All patients who were included in this study underwent curative resection for their primary tumors, according to surgical oncological principles, and total mesorectal excision was performed by experienced surgical experts. All regional lymph nodes were dissected, including IMA lymph nodes. The inferior mesenteric vein was ligated and divided. Distal surgical margins of $>5 \mathrm{~cm}$ were achieved for tumors located above the peritoneal reflection, and surgical margins were $>2 \mathrm{~cm}$ for tumors located below the peritoneal reflection. Lateral pelvic lymph node dissection was not performed routinely in the surgical procedure. The primary tumor and all dissected lymph node specimens were stained with hematoxylin and eosin and evaluated by experienced pathologists.

\subsection{Statistical Analysis}

Statistical analysis was performed using the SPSS 17.0. With lymph node metastasis as an end event, single factor and multiple factors Logistic regression model is established. We used $x^{2}$ tests to analyze the lymph node metastasis related factors and used multiariable Logistic regression analysis to analyze the single factor analysis meaningful variables. 


\section{Results}

A total of 4357 lymph nodes were detected in 258 patients with rectal cancer, with an average of 16.90 lymph nodes per case. The number of lymph nodes detected in each patient was greater than or equal to 12 cases. There were 92 patients with lymph node metastasis. For the distance from the lower margin of rectal tumor to the anal margin, the circumference, gross type and depth of invasion, the accuracy of rectal finger examination is shown in Tables 1-4. Univariate analysis showed that there was no significant difference between lymph node metastasis and the distance from the lower margin of the tumor to the anal margin, but there have significant difference between lymph node metastasis and circumference, gross types as well as depth of infiltration (Tables 5-8). Multivariate analysis showed that gross type and depth of invasion were independent factors affecting lymph node metastasis (Table 9).

Table 1. Comparison of the accuracy rate of digitalexamination and postoperative pathology: the anal margin to the lower margin of the tumor.

\begin{tabular}{ccccccc}
\hline & $3 \mathrm{~cm}$ & $4 \mathrm{~cm}$ & $5 \mathrm{~cm}$ & $6 \mathrm{~cm}$ & $7 \mathrm{~cm}$ & $8 \mathrm{~cm}$ \\
\hline $\mathrm{DE}(\mathrm{n})$ & 38 & 45 & 61 & 43 & 39 & 32 \\
$\operatorname{PPJ}(\mathrm{n})$ & 36 & 40 & 53 & 34 & 30 & 22 \\
$\begin{array}{c}\text { Accuracy } \\
(\%)\end{array}$ & 94.74 & 88.89 & 86.89 & 79.07 & 76.92 & 68.75 \\
\hline
\end{tabular}

DE: Digital examination; PPJ: postoperative pathological judgment.

Table 2. Comparison of the accuracy rate of digital examination and postoperative pathology: the circulus degree.

\begin{tabular}{ccccc}
\hline & $1 / 4 \geq \mathrm{T}$ & $2 / 4 \geq \mathrm{T}>1 / 4$ & $3 / 4 \geq \mathrm{T}>2 / 4$ & $\mathrm{~T}>3 / 4$ \\
\hline DE (n) & 59 & 65 & 66 & 68 \\
PPJ (n) & 51 & 55 & 55 & 58 \\
$\begin{array}{c}\text { Accuracy } \\
\text { (\%) }\end{array}$ & 86.44 & 84.61 & 83.33 & 85.29 \\
\hline
\end{tabular}

DE: Digital examination; PPJ: postoperative pathological judgment.

Table 3. Comparison of the accuracy rate of digital examination and postoperative pathology: gross pathologic type.

\begin{tabular}{ccccc}
\hline & UT & IDU & IU & ID \\
\hline DE (n) & 52 & 85 & 78 & 43 \\
PPJ (n) & 44 & 73 & 68 & 36 \\
$\begin{array}{c}\text { Accuracy } \\
(\%)\end{array}$ & 84.62 & 85.88 & 87.18 & 83.72 \\
\hline
\end{tabular}

DE: Digital examination; PPJ: postoperative pathological judgment; UT: uplift type; IDU: local defined ulcer; IU: Infiltrating ulcer; ID: Infiltrating diffuse. 
Table 4. Comparison of the accuracy rate of digital examination and postoperative pathology: for the infiltration depth.

\begin{tabular}{ccccc}
\hline & M\&S & ML & IWO & IV \\
\hline DE (n) & 16 & 42 & 157 & 43 \\
$\operatorname{PPJ}(\mathrm{n})$ & 13 & 37 & 139 & 36 \\
Accuracy (\%) & 81.25 & 88.10 & 88.54 & 83.72 \\
\hline
\end{tabular}

DE: Digital examination; PPJ: postoperative pathological judgment; M\&S: mucosa and submucosa; ML: muscular layer; IWO: Intestinal wall outside; IV: infringe viscera.

Table 5. The Digital examination vspostoperative specimens of lymph node metastasis compared in the distance from rectal cancer's inferior border to anal.

\begin{tabular}{|c|c|c|c|c|c|c|c|c|c|c|c|c|c|}
\hline & \multirow{2}{*}{$\mathrm{n}$} & \multicolumn{6}{|c|}{$\begin{array}{c}\text { Digital } \\
\text { examination }\end{array}$} & \multicolumn{6}{|c|}{$\begin{array}{c}\text { Postoperative } \\
\text { pathologic examination }\end{array}$} \\
\hline & & $3 \mathrm{~cm}$ & $4 \mathrm{~cm}$ & $5 \mathrm{~cm}$ & $6 \mathrm{~cm}$ & $7 \mathrm{~cm}$ & $8 \mathrm{~cm}$ & $3 \mathrm{~cm}$ & $4 \mathrm{~cm}$ & $5 \mathrm{~cm}$ & $6 \mathrm{~cm}$ & $7 \mathrm{~cm}$ & $8 \mathrm{~cm}$ \\
\hline $\mathrm{LN}(+)$ & 92 & 12 & 14 & 24 & 14 & 16 & 12 & 11 & 15 & 25 & 15 & 16 & 10 \\
\hline $\mathrm{LN}(-)$ & 166 & 26 & 31 & 37 & 29 & 23 & 20 & 27 & 31 & 37 & 32 & 24 & 15 \\
\hline \multicolumn{2}{|c|}{$\mathrm{x}^{2}$ tests } & \multicolumn{6}{|c|}{$\mathrm{x}^{2}=1.854 \mathrm{p}>0.05$} & \multicolumn{6}{|c|}{$\mathrm{x}^{2}=2.032 \mathrm{p}>0.05$} \\
\hline
\end{tabular}

LN: lymph node.

Table 6. The Digital examination vs postoperative specimens of lymph node metastasis compared in circulus degree.

\begin{tabular}{|c|c|c|c|c|c|c|c|c|c|}
\hline & \multirow{2}{*}{$\mathrm{n}$} & \multicolumn{4}{|c|}{$\begin{array}{c}\text { Digital } \\
\text { examination }\end{array}$} & \multicolumn{4}{|c|}{$\begin{array}{c}\text { Postoperative } \\
\text { pathologic examination }\end{array}$} \\
\hline & & \multicolumn{8}{|c|}{$1 / 4 \geq \mathrm{T} 2 / 4 \geq \mathrm{T}>1 / 43 / 4 \geq \mathrm{T}>2 / 4 \mathrm{~T}>3 / 41 / 4 \geq \mathrm{T} 2 / 4 \geq \mathrm{T}>1 / 43 / 4 \geq \mathrm{T}>2 / 4 \mathrm{~T}>3 / 4$} \\
\hline $\mathrm{LN}(+)$ & 92 & 10 & 19 & 28 & 35 & 9 & 19 & 31 & 33 \\
\hline $\mathrm{LN}(-)$ & 166 & 49 & 46 & 38 & 33 & 47 & 51 & 38 & 30 \\
\hline \multicolumn{2}{|c|}{$\mathrm{x}^{2}$ tests } & \multicolumn{4}{|c|}{$\mathrm{x}^{2}=3.839, \mathrm{p}<0.05$} & \multicolumn{4}{|c|}{$\mathrm{x}^{2}=3.695, \mathrm{p}<0.05 \mathrm{i}$} \\
\hline
\end{tabular}

LN: lymph node.

Table 7. The Digital examination vspostoperative specimens of lymph node metastasis compared in gross pathologic type.

\begin{tabular}{|c|c|c|c|c|c|c|c|c|c|}
\hline & \multirow{2}{*}{$\mathrm{n}$} & \multicolumn{4}{|c|}{$\begin{array}{c}\text { Digital } \\
\text { examination }\end{array}$} & \multicolumn{4}{|c|}{$\begin{array}{c}\text { Postoperative } \\
\text { pathologic examination }\end{array}$} \\
\hline & & UT & IDU & IU & IDUT & UT & IDU & IU & IDUT \\
\hline $\mathrm{LN}(+)$ & 92 & 10 & 19 & 26 & 37 & 8 & 19 & 27 & 38 \\
\hline $\mathrm{LN}(-)$ & 166 & 42 & 66 & 52 & 6 & 40 & 67 & 56 & 3 \\
\hline \multicolumn{2}{|c|}{$\mathrm{x}^{2}$ tests } & \multicolumn{4}{|c|}{$\mathrm{x}^{2}=6.651, \mathrm{p}<0.01$} & \multicolumn{4}{|c|}{$\mathrm{x}^{2}=7.572, \mathrm{p}<0.01$} \\
\hline
\end{tabular}

UT: uplift type; IDU: local defined ulcer; IU: Infiltrating ulcer; ID: Infiltrating diffuse. LN: lymph node. 
Table 8. The Digital examination vspostoperative specimens of lymph node metastasis compared in infiltration depth.

\begin{tabular}{|c|c|c|c|c|c|c|c|c|c|}
\hline & \multirow[t]{2}{*}{$\mathrm{n}$} & \multicolumn{4}{|c|}{$\begin{array}{c}\text { Digital } \\
\text { examination }\end{array}$} & \multicolumn{4}{|c|}{$\begin{array}{c}\text { Postoperative } \\
\text { pathologic examination }\end{array}$} \\
\hline & & $M \& S$ & ML & IWO & IV & $\mathrm{M} \& \mathrm{~S}$ & ML & IWO & IV \\
\hline $\mathrm{LN}(+)$ & 92 & 0 & 6 & 43 & 43 & 0 & 11 & 34 & 47 \\
\hline $\mathrm{LN}(-)$ & 166 & 16 & 36 & 114 & 0 & 15 & 36 & 115 & 0 \\
\hline \multicolumn{2}{|c|}{$\mathrm{x}^{2}$ tests } & \multicolumn{4}{|c|}{$\mathrm{x}^{2}=14.354, \mathrm{p}<0.01$} & \multicolumn{4}{|c|}{$x^{2}=16.586, p<0.01$} \\
\hline
\end{tabular}

M\&S: mucosa and submucosa; ML: muscular layer; IWO: Intestinal wall outside; IV: infringe viscera; LN: lymph node.

Table 9. Lymph node metastasis multi-factor analyzed with rectal cancer characteristics through digital examination.

\begin{tabular}{ccccc}
\hline Variate & PRC & SE & RR & p-value \\
\hline circulus degree & 0.261 & 0.112 & 1.282 & 0.094 \\
macroscopic pathology & 0.232 & 0.107 & 1.677 & 0.035 \\
infiltration depth & 0.367 & 0.106 & 1.358 & 0.011 \\
\hline
\end{tabular}

PRC: partial regression coefficient; SE: standard error; RR: relative risk.

\section{Discussions}

Most rectal cancer occurs in the lower rectum, and about $80 \%$ of them are located below the peritoneal reflex plane, within the range of digital rectal examination. Through digital rectal examination, on the one hand, rectal cancer can be diagnosed and misdiagnosis can be avoided. On the other hand, the degree of progression of rectal cancer can be assessed initially to provide further guidance for further examination. Correct digital rectal examination, in addition to being able to assess the distance from the lower edge of the tumor to the anal margin, the invasion circumference of the intestinal wall of the tumor, the general type, but also indirectly determine the depth of tumor invasion, with or without intestinal obstruction and lesions outside the rectal wall. In this study, we chose a rectal cancer within $8 \mathrm{~cm}$ from the lower edge of the tumor to the anal margin. The results showed that the distance from the lower edge of the tumor to the anal margin determined by digital rectal examination was compared with the results of postoperative pathological examination. The accuracy rate was $68.75 \%$ to $94.74 \%$. The farther the distance from the anal margin was, the greater the error was. The reason for this may be, that the rectum is not perpendicular, and that the sacrum depression is curved and can be extended after surgery, so that the tumor is farther away from the anal margin.

As long as you take proper and careful fingertip palpation, you should have a higher accuracy rate in the invasion circumference of the intestinal wall and the gross pathological type of tumor. The accuracy rate was $83.33 \%-86.44 \%$ and $83.72 \%-87.18 \%$ respectively in our study. In imaging, localized lesions are indistinguishable from fibrosis and tumor invasion. Although the diagnosis of ad- 
vanced T3 tumors is relatively easy, the distinction between T2 tumors and early T3 tumors requires considerable experience and high-quality images [10]. As the invasion of the intestinal wall increases, the chance of tumor metastasis increases. Tumor growth not only grows along the surface of the intestinal wall, but also penetrates into the intestinal wall. Therefore, the larger the diameter of the tumor, its depth will inevitably increase, and the chance of invading lymph nodes will increase. Different biological characteristics of rectal cancer have different growth patterns and invasiveness, and have different effects on lymph node metastasis. The intestinal muscularis and serosal layer play a role in the resistance to cancer cell invasion. As the tumor infiltrates deep into the intestinal wall and outside the intestinal wall, the larger the contact area with the lymphatic vessels, the greater the chance of lymph node metastasis. Depth of tumor invasion outside the muscularispropria is not considered in TNM staging; however, it has substantial clinical significance. The American Joint Committee on Cancer criteria for the staging of rectal cancer has been modified to incorporate depth of invasion outside the muscularispropria. The majority $(80 \%)$ of rectal tumors are stage T3 lesions that form a heterogeneous group, with 5 -year survival rates varying based on the depth of tumor invasion outside the muscularispropria. The cancer-specific survival rate drops from $85 \%$ to $54 \%$, independent of nodal involvement, when the depth of tumor invasion outside the muscularispropria exceeds $5 \mathrm{~mm}$ [14]. In the subgroup of superficial tumors, EUS may be more accurate in deciding whether the tumour has penetrated the rectal wall [15], EUS is far from being ideal in identifying regional lymph node metastases. The ability of differentiating tumor invasion range was poor [16]. In addition, the examination of patients with intestinal stenosis or high rectal tumors was significantly restricted [17]. By palpation at the fingertips, the accuracy we perceive was $81.25 \%$ - 88.54\%.

Because of the application of modern advanced examination equipment, many doctors ignore digital rectal examination in preoperative evaluation of rectal cancer value. They think rectal finger diagnosis is of little significance before operation. Accurately assessing lymph node metastasis before surgery is directly related to the development of treatment plans and the choice of surgical procedures, as well as preconditioning, and it is important to improve the quality of the patient's life [18]. Our univariate analysis showed that the invasion circumference of the intestinal wall of the tumor, the gross pathological type, and the depth of invasion were all related to the positive rate of lymph nodes, but further multivariate analysis showed that the gross pathological types and depth of invasion were two important factors affecting lymph node metastasis.

\section{Conclusion}

In conclusion, by correct and detailed digital rectal examination before surgery, palpation through your fingertips, you will perceive the high accuracy of lymph node metastasis in rectal cancer. It is necessary to combine the palpation of fin- 
gertips with the visual inspection of imaging to perceive the preoperative staging of low and middle rectal cancer. Although the limitation of this study is that surgeons who perform preoperative anal finger examinations require clinically experienced, longer index fingers.

\section{Conflicts of Interest}

The authors declare no conflicts of interest regarding the publication of this paper.

\section{References}

[1] Heald, R.J., Moran, B.J., Ryall, R.D., Sexton, R. and MacFarlane, J.K. (1998) Rectal Cancer: The Basingstoke Experience of Total Mesorectal Excision, 1978-1997. The Archives of Surgery, 133, 894-899. https://doi.org/10.1001/archsurg.133.8.894

[2] Kusano, T., Inomata, M., Hiratsuka, T., et al. (2014) A Comparison of Laparoscopic and Open Surgery Following Pre-Operative Chemoradiation Therapy for Locally Advanced Lower Rectal Cancer. Japanese Journal of Clinical Oncology, 44, 305-310. https://doi.org/10.1093/jjco/hyu013

[3] Engstrom, P.F., Arnoletti, J.P., Benson, A.B., et al. (2009) NCCN Clinical Practice Guidelines in Oncology: Rectal Cancer. Journal of the National Comprehensive Cancer Network, 7, 838-848.

[4] Sauer, R., Becker, H., Hohenberger, W., et al. (2004) Preoperative versus Postoperative Chemoradiotherapy for Rectal Cancer. The New England Journal of Medicine, 351, 1731-1740. https://doi.org/10.1056/NEJMoa040694

[5] van Gijn, W., Marijnen, C.A., Nagtegaal, I.D., et al. (2011) Preoperative Radiotherapy Combined with Total Mesorectal Excision for Resectable Rectal Cancer: 12-Year Follow-Up of the Multicentre, Randomised Controlled TME Trial. The Lancet Oncology, 12, 575-582. https://doi.org/10.1016/S1470-2045(11)70097-3

[6] Jess, P., Seiersen, M., Ovesen, H., et al. (2014) Has PET/CT a Role in the Characterization of Indeterminate Lung Lesions on Staging CT in Colorectal Cancer? A Prospective Study. European Journal of Surgical Oncology, 40, 719-722. https://doi.org/10.1016/j.ejso.2013.11.030

[7] Beets-Tan, R.G., Beets, G.L., Vliegen, R.F., et al. (2001) Accuracy of Magnetic Resonance Imaging in Prediction of Tumour-Free Resection Margin in Rectal Cancer Surgery. The Lancet, 357, 497-504. https://doi.org/10.1016/S0140-6736(00)04040-X

[8] Blomqvist, L., Machado, M., Rubio, C., et al. (2000) Rectal Tumour Staging: MR Imaging Using Pelvic Phasedarray and Endorectal Coils vs. Endoscopic Ultrasonography. European Radiology, 10, 653-660. https://doi.org/10.1007/s003300050979

[9] Okafor, P.N., Swanson, K., Shah, N. and Talwalkar, J.A. (2018) Endoscopic Ultrasound for Rectal Cancer Staging: A Population-Based Study of Utilization, Impact on Treatment Patterns, and Survival. Journal of Gastroenterology and Hepatology, $1,26$.

[10] Beets-Tan, R.G. and Beets, G.L. (2004) Rectal Cancer: Review with Emphasis on MR Imaging. Radiology, 232, 335-346. https://doi.org/10.1148/radiol.2322021326

[11] Waizer, A., Zitron, S., Ben-Baruch, D., Baniel, J., Wolloch, Y. and Dintsman, M. (1989) Comparative Study for Preoperative Staging of Rectal Cancer. Diseases of the Colon \& Rectum, 32, 53-56. https://doi.org/10.1007/BF02554726

[12] Kaur, H., Choi, H., You, Y.N., et al. (2012) MR Imaging for Preoperative Evaluation 
of Primary Rectal Cancer: Practical Considerations. Radiographics, 32, 389-409. https://doi.org/10.1148/rg.322115122

[13] Halefoglu, A.M., Yildirim, S., Avlanmis, O., Sakiz, D. and Baykan, A. (2008) Endorectal Ultrasonography versus Phased-Array Magnetic Resonance Imaging for Preoperative Staging of Rectal Cancer. World Journal of Gastroenterology, 14, 3504-3510. https://doi.org/10.3748/wjg.14.3504

[14] Merkel, S., Mansmann, U., Siassi, M., Papadopoulos, T., Hohenberger, W. and Hermanek, P. (2001) The Prognostic in Homogeneity in pT3 Rectal Carcinomas. International Journal of Colorectal Disease, 16, 298-304. https://doi.org/10.1007/s003840100309

[15] Mortensen, N. (1992) Rectal and anal Endosonography. Gut, 33, 148-149. https://doi.org/10.1136/gut.33.2.148

[16] Rafaelsen, S.R., Kronborg, O. and Fenger, C. (1994) Digital Rectal Examination and Transrectal Ultrasonography in Staging of Rectal Cancer: A Prospective, Blind Study. Acta Radiologica, 35, 300-304. https://doi.org/10.3109/02841859409172387

[17] Schizas, A.M., Williams, A.B. and Meenan, J. (2009) Endosonographic Staging of Lower Intestinal Malignancy. Best Practice \& Research: Clinical Gastroenterology, 23, 663-670. https://doi.org/10.1016/j.bpg.2009.06.006

[18] Bellows, C.F., Jaffe, B., Bacigalupo, L., Pucciarelli, S. and Gagliardi, G. (2011) Clinical Significance of Magnetic Resonance Imaging Findings in Rectal Cancer. World Journal of Radiology, 3, 92-104. https://doi.org/10.4329/wjr.v3.i4.92 